\title{
NOTAS SOBRE LA BIOLOGÍA Y PESCA DEL ZÚNGARO TIGRINUS Brachyplatystoma tigrinum (Britski, 1981) EN EL RíO AMAZONAS, PERÚ
}

\author{
Fernando ALCÁNTARA ${ }^{1}$, Fred CHU ${ }^{1}$, Luciano RODRIGUEZ ${ }^{1}$, Carlos CHÁVEZ ${ }^{1}$, Alfonso BERNUY ${ }^{1}$, \\ Teddy BARBARÁN ${ }^{1}$, Salvador TELLO ${ }^{1}$, Germán MURRIETA ${ }^{2}$, Carmen GARCÍA-DÁVILA ${ }^{1}$, Jesús \\ NUÑEZ ${ }^{3}$
}

1 Instituto de Investigaciones de la Amazonía Peruana, IIAP. Programa para el Uso y Conservación del Agua y sus Recursos (AQUAREC), Carretera Iquitos - Nauta, Km 4.5. Iquitos, Perú, E.mail : fab_001@hotmail.com

2 Universidad Nacional de la Amazonía Peruana - UNAP. Escuela de Post Grado. Cátedra CONCYTEC. Maestría en Acuicultura. Iquitos, Perú.

3 Institut de Recherche pour le Développement-IRD. 175 Caviar, BP 5095, 34196 Montpellier cedex 05, France.

\begin{abstract}
RESUMEN
El zúngaro tigrinus Brachyplatystoma tigrinum es un pez Pimelodido que habita principalmente las aguas lóticas de los grandes ríos neotropicales entre ellos el Amazonas, el Marañón y el Ucayali. Los alevinos de B. tigrinum son un componente importante del mercado internacional de peces ornamentales, y los adultos se consumen localmente. La mayoría de la pesca se realiza con redes, que varían en tamaño (de 300 x 15 brazos largos para los peces adultos y redes bolicheras de $1 / 4$ de pulgada de malla, de 40 a $50 \mathrm{~m}$ de longitud y dos a tres brazas para alevinos). La captura se realiza con redes de diversos tamaños ( 300 x 15 brasas para peces adultos). La relación entre la longitud y el peso del zúngaro tigrinus adulto, recientemente capturado, responde a la ecuación $\mathrm{W}(\mathrm{t})=$ $0.0034433 \mathrm{Lt}^{3.19027953}(\mathrm{r}=0.98283632)$, lo que indica una tendencia de crecimiento isométrico, la especie presenta un factor de condición de $0,77 \pm 0,12$. La reproducción tiene lugar al final de la temporada de vaciante de las aguas bajas y comienzo de la creciente; cerca de Iquitos, esto ocurre entre noviembre y febrero. La reproducción tiene lugar al final de la temporada de vaciante de las aguas bajas y comienzo de la creciente; cerca de Iquitos, esto ocurre entre noviembre y febrero. Cuando se cultiva en estanques de agua negra, B. tigrinum mostró una alta incidencia del parasitismo Argulus pestifer (crustácea). Aspectos fundamentales de la biología de esta especie son aun desconocidos, incluida la conducta de alimentación y reproducción, así como las interacciones con los parásitos y patógenos.
\end{abstract}

PALABRAS CLAVE: Zúngaro tigrinus, Brachyplatystoma tigrinum, bioecología, pesca, parasitismo

\section{NOTES ON THE BIOLOGY AND RESOURCE STATUS OF ZÚNGARO TIGRINUS Brachyplatystoma tigrinum (Britski, 1981) IN THE AMAZON RIVER, PERU}

\begin{abstract}
The zúngaro tigrinus (Brachyplatystoma tigrinum) is a Pimelodid fish that inhabits mainly lotic waters of large neotropical rivers including the Amazon, Marañon and Ucayali. The fry of B. tigrinum are an important constituent of the international ornamental fish market, and the adults are consumed locally. Most fishing is accomplished with nets, which vary in size (from $300 \times 15$ arm-lengths for adult fish to seine nets of $1 / 4$ inch mesh, 40 to $50 \mathrm{~m}$ long and 2 to 3 coals for juveniles). The relationship between length and weight of fresh-captured $\mathrm{B}$. tigrinum is represented by the equation $\mathrm{W}(\mathrm{t})=0.0034433 \mathrm{Lt}^{3.19027953}(\mathrm{r}=0.98283632)$, which indicates an isometric growth trend; the species exhibits a condition factor of $0.77 \pm 0.12$. Reproduction occurs at the end of the low-water season and the beginning of high-water; near Iquitos, this occurs between November and February. When grown in black water ponds, B. tigrinum showed a high incidence of parasitism Argulus (Crustacea). Fundamental aspects of this species' biology remain unknown, including feeding- and breeding behavior, as well as interactions with parasites and pathogens.
\end{abstract}

KEYWORDS: Zúngaro tigrinus, Brachyplatystoma tigrinum, bio-ecology, fishing, riding. 


\section{INTRODUCCIÓN}

La explotación de peces para el comercio ornamental internacional es un problema de conservación cada vez más grande (Moreau \& Coomes, 2007). Los peces de acuario son las mascotas más populares del mundo y generan una industria creciente; de ellos, cerca del $90 \%$ son de agua dulce. Aunque la mayor parte del comercio de peces de agua dulce en el mundo proviene de cultivo, las especies amazónicas son casi exclusivamente extraídas directamente de los ambientes naturales (Olivier, 2001). El comercio ornamental ya contribuyó a la extinción de varias poblaciones locales de peces en el sudeste asiático y probablemente a caídas en el Brasil (ej. pez disco). En el Perú, el mercado que estaba basado en exportaciones masivas de especies de bajo valor comercial, ha cambiado recientemente hacia especies de alto valor como la arahuana, algunas rayas, loricáridos y algunos grandes pimelódidos como $B$. tigrinum. De hecho, este pez que alcanza el mayor precio de exportación por unidad después de la raya tigre llegando en ciertos casos a costar entre 300 a 600 euros cada uno y su demanda es creciente en los mercados asiáticos (Moreau \& Coomes, 2007). Lo poco que se conocía de la especie hasta antes de esta publicación era que al estado adulto, vive en el agua corriente de los grandes ríos, en la Amazonía peruana, en especial; en la zona cóncava de los meandros, en la que predomina la erosión y la consiguiente migración lateral del río. Sin embargo, existe la necesidad de mejorar el conocimiento sobre biología y ecología de esta importante especie, en orden a contribuir a su uso sostenible en la Amazonía peruana.

\section{MATERIAL Y MÉTODOS}

Sobre la base de los conocimientos tradicionales del zúngaro tigrinus, reportados por Chávez et al. (2008), fueron efectuadas observaciones de las características del hábitat, de las zonas de pesca, calidad de agua, aparejos de pesca, captura, épocas de pesca; así como de algunas de las características biológicas del zúngaro tigrinus Brachyplatystoma tigrinum.

Las observaciones fueron efectuadas en el río Amazonas, en el sector comprendido entre las coordenadas geográficas S $03^{\circ} 55^{\prime} 21.5^{\prime \prime}$ - W $73^{\circ} 09^{\prime} 24.6^{\prime \prime}$ y S $03^{\circ} 41^{\prime} 51.0^{\prime \prime}$ W $73^{\circ} 09^{\prime} 21.5^{\prime \prime}$, complementadas con observaciones de cría en estanques de las instalaciones del Centro de Investigaciones de Quistococha del Instituto de Investigaciones de la Amazonía Peruana.

\section{RESULTADOS Y DISCUSIÓN}

\section{CARACTERÍSTICAS GENERALES}

El zúngaro tigrinus Brachyplatystoma tigrinum es un pez que pertenece al grupo de los grandes bagres o Pimelodidos que, al estado adulto, puede alcanzar hasta $85 \mathrm{~cm}$ de longitud, con un peso de 5,200 g. Su coloración general, al estado adulto, es negruzca, con bandas blanquecinas que se extienden en forma oblicua al eje longitudinal del cuerpo $\mathrm{y}$, en número variable de 13 a 15 (Figura 1A). El patrón de coloración descrito, sin embargo, varía a través del desarrollo del zúngaro tigrinus ya que, al estado de alevino ( 2 a $5 \mathrm{~cm}$ de longitud), presenta una coloración general blanquecina a rojiza, sin las bandas blanquecinas de los adultos. (Figura 1B).

El zúngaro tigrinus tiene un cuerpo fusiforme, ligeramente plano en la región ventral. Su cabeza es grande y ligeramente achatada, los ojos son pequeños y de posición dorsal, la boca es grande y de posición ventral, rodeada de seis barbillas filamentosas, de las cuales, dos son maxilares y cuatro son mentonianas. La aleta dorsal es corta y ubicada en el tercio anterior del cuerpo. La aleta adiposa es también corta y ubicada en el tercio posterior del cuerpo, sobre el pedúnculo caudal. Entre las aletas pares presenta las pectorales y las ventrales de desarrollo moderado. Adicionalmente, el zúngaro tigrinus presenta una aleta ubicada, ventralmente, en la región del pedúnculo caudal, a modo de quilla. La aleta caudal es bilobulada y cada lóbulo termina en un largo filamento que el zúngaro tigrinus agita suavemente formando ondulaciones a medida que se desplaza en el agua. Los filamentos de la aleta caudal, están presentes de preferencia en los alevinos y juveniles y son menos frecuentes en los adultos.

En la boca se observa la presencia de dientes pequeños y numerosos, dispuestos en bandas o placas, como son, la placa maxilar y la placa mandibular (Figura 2A). En la región faríngea se observan cuatro arcos branquiales y, en el techo de la faringe se observa dos formaciones carnosas con numerosos y pequeños dientes, constituyendo las placas masticatrices o almohadillas faríngeas que se corresponden con la placa faríngea inferior (Figura 2B).

El tubo digestivo es corto, como ocurre en los peces de régimen alimenticio carnívoro. El estómago es relativamente grande y dilatable, en forma de V. En el techo de la cavidad celomática presenta una vejiga de naturaleza serosa, protegida de tejido membranoso y cubriendo ventralmente la vejiga se encuentra una formación de tejido adiposo sobre la que se ubican las gónadas. 

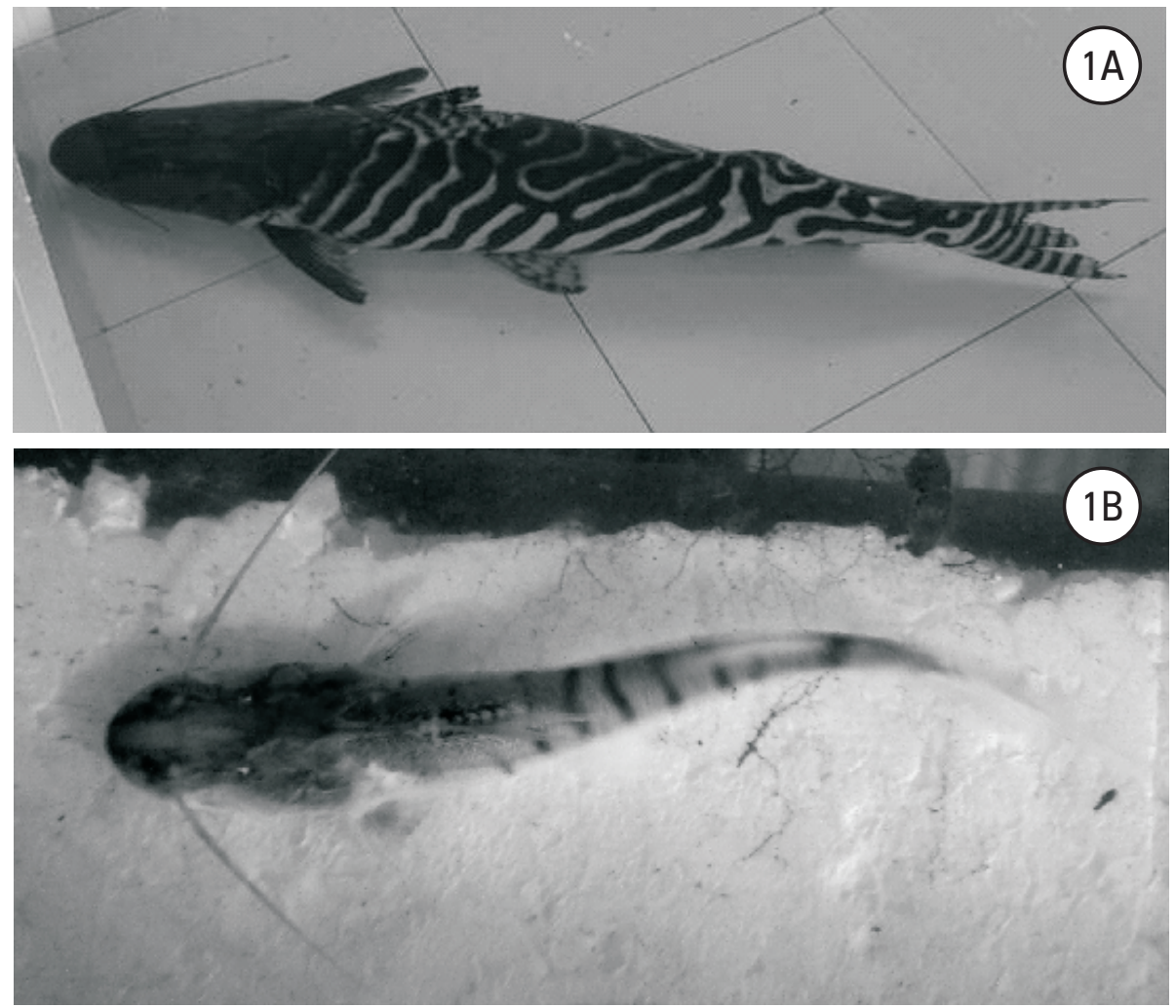

Figura 1. Zúngaro tigrinus Brachyplatystoma tigrinum: 1A especimen adulto $(85 \mathrm{~cm}), 1 B$ Alevino $(7 \mathrm{~cm})$ capturados en el rio Amazonas.
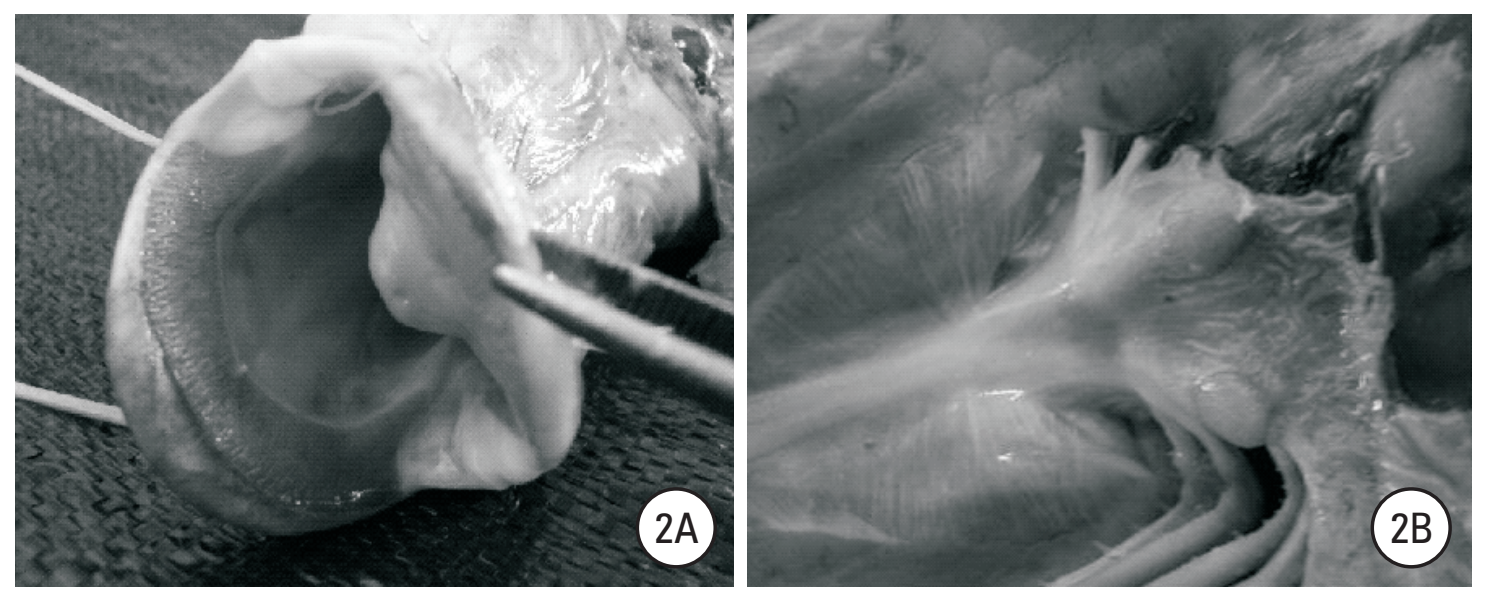

Figura 2. $2 \mathrm{~A}$ placa dentaria; $2 \mathrm{~B}$ almohadillas faríngeas $\mathrm{y}$ arcos branquiales del zúngaro tigrinus Brachyplatystoma tigrinum. 


\section{CARACTERÍSTICAS DEL HÁBITAT}

El zúngaro tigrinus al estado de alevino, juvenil y adulto vive en aguas lóticas de los grandes ríos, como el Amazonas, en especial, en las zonas de costa brava, en las que predomina la erosión de la orilla externa y es frecuente la presencia de palizadas. Sin embargo, esto podría deberse simplemente, a selectividad involuntaria de las áreas de pesca por parte de los pescadores, sin que signifique preferencia de hábitat de la especie. El hábitat se caracteriza por presentar agua de color blanco, con alta disponibilidad de sólidos en suspensión, baja transparencia, que puede llegar a $30 \mathrm{~cm}$, alta disponibilidad de oxígeno disuelto (5 mg/l), temperatura alrededor de $27^{\circ} \mathrm{C}$ y pH 7 .

En individuos manejados en estanques de agua negra, eventualmente, se observó comportamiento territorial consistente en el establecimiento de una zona, de unos $0.80 \mathrm{~m}$ de diámetro, ubicada en la zona litoral del estanque, a unos $0.60 \mathrm{~m}$ de profundidad, libre de algas y sedimentos; en cuyo centro permanece el zúngaro tigrinus por largos periodos.

\section{ZONAS DE PESCA}

Las zonas de pesca del zúngaro tigrinus en la región Loreto, están restrictas al río Amazonas, en las cercanías de la ciudad de Iquitos (Figura 3), sea que se trate de adultos o de alevinos. En el tramo comprendido entre Gran Perú, cerca de la localidad de

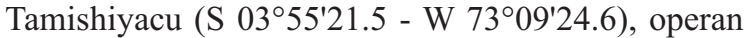
principalmente los pescadores de tigrinus adultos $\mathrm{y}$, en las inmediaciones de la comunidad de Santa Rosa, aguas abajo de la refinería Luis F. Díaz (S 0341'51.0' W 7309'21.5), operan los pescadores de alevinos. Sin embargo, Se registró también la presencia de esta especie cerca del Pongo de Manseriche, en el río Marañón(Sánchez, 2008).

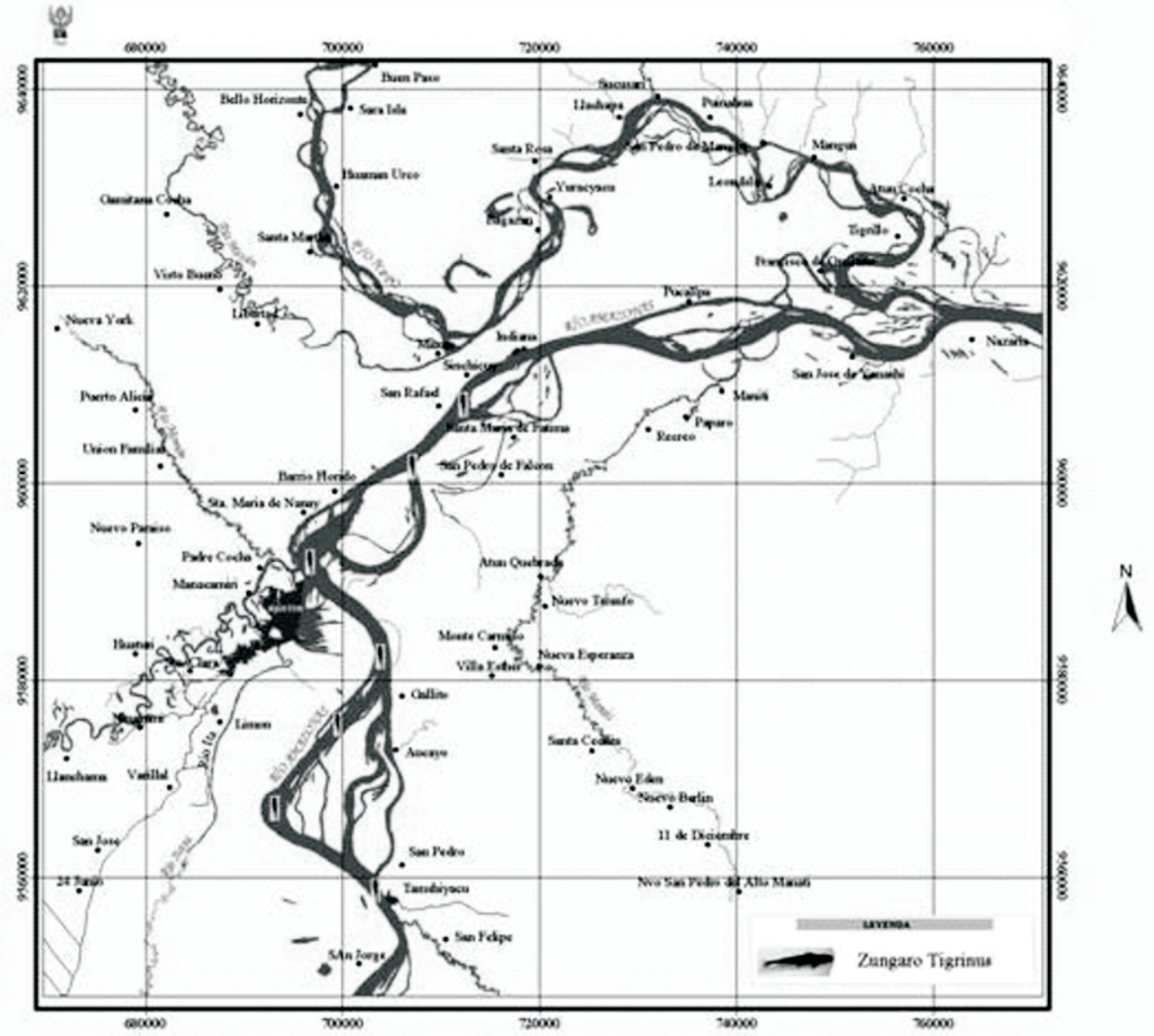

Figura 3. Zonas de pesca comercial del zúngaro tigrinus Brachyplatystoma tigrinum en la región de Loreto en la Amazonía peruana. 


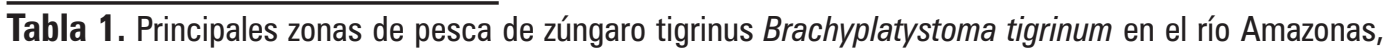
Amazonía peruana.

\begin{tabular}{|l|c|c|}
\hline \multicolumn{1}{c|}{ LUGAR } & $\begin{array}{c}\text { LONGITUD PARA } \\
\text { LA PESCA (m) }\end{array}$ & $\begin{array}{c}\text { TIEMPO DESDE } \\
\text { IQUITOS (horas) }\end{array}$ \\
\hline Frente al caserío de Aucayo & 450 & 3.0 aguas arriba \\
\hline Frente al caserío de Gallito & 350 & 2.0 aguas arriba \\
\hline Frente a Padre Isla & 250 & 1.0 aguas arriba \\
\hline Frente al caserío de Panguana Zona I y II & 500 & 3.5 aguas arriba \\
\hline Timareo & 500 & 1.5 aguas arriba \\
\hline Frente al campo experimental del INIA & 500 & 1.0 aguas arriba \\
\hline Frente a Masusa. Boca del río Itaya & 300 & 0.5 aguas abajo \\
\hline Frente a la base del SIMAI & 400 & 2.5 aguas abajo \\
\hline Frente al caserío Santa Rosa & 400 & 3.0 aguas abajo \\
\hline
\end{tabular}

Fuente: APPOLI, 2009

Los alevinos son capturados por pescadores de peces ornamentales, en tramos cortos de aguas someras que corresponden a brazos secundarios del río que, en vaciante, corresponden a playas o barriales de cultivo de arroz. Mientras que, los juveniles y adultos son capturados por pescadores de peces de consumo, en el curso de la corriente del río. Las principales zonas de pesca, están ubicadas en un radio de 1:00 a 3:00 horas aguas arriba y aguas abajo de la ciudad de Iquitos. Estas zonas de pesca varían también en su extensión a lo largo del río (Tabla 1). Según registros de pesca de APPOLI 2009, Las zonas con mayores volúmenes de captura de alevinos de zúngaro tigrinus en el periodo comprendido entre diciembre del 2008 a mayo del 2009 fueron Timareo (2100 ejemplares) seguido de Santa Rosa (1400 ejemplares).

\section{APAREJOS DE PESCA Y TURNOS DE PESCA}

Los pescadores de peces de consumo utilizan redes de $300 \times 1.5$ brazas, con lastres de plomo y flotadores de material sintético, malla de 6 pulgadas, hilo de nylon número $36 \mathrm{y}$ color verde. Estas redes son operadas a la deriva por 24 horas, en turnos de 12 horas, cada uno, de 06:00 a 18:00 horas y de 18:00 a 06:00 horas. Estas redes son operadas en el curso de la corriente del río, maniobrando con una o dos embarcaciones equipadas con motor fuera de borda (denominados localmente "peque peque") para orientar su desplazamiento en la corriente del río. Para la faena de pesca cada embarcación lleva dos pescadores y realiza lances de
15 a 30 minutos, luego de la cual la red es recuperada. Los pescadores dirigen la red con la embarcación para evitar enredarse en las palizadas sumergidas, que puede significar la pérdida total del material de pesca.

La captura de los alevinos del zúngaro tigrinus se realiza con redes bolicheras de 40 a $50 \mathrm{~m}$ de longitud y dos a tres brazas de de ancho y de $1 / 4$ de pulgada de malla, provistas de una línea de flotadores y una línea de lastre de 40 a $50 \mathrm{~kg}$ de peso. La modalidad de captura consiste en lanzar la red en la corriente, en las zonas someras del río, maniobrando desde la embarcación principal y la embarcación auxiliar, con la ayuda de motores fuera de borda. Cada embarcación lleva tres o cuatro operadores para realizar la faena de pesca. El recorrido del lance es de 5 a 10 minutos y luego recuperan la red.

La pesca de los alevinos se puede practicar solamente en zonas poco profundas con fondos llanos y sin palizadas. Esta actividad, es realizada principalmente en las primeras horas del día, comenzando entre las cinco y seis hasta las ocho o nueve de la mañana. Un segundo turno se realiza al atardecer comenzando a las tres o cuatro de la tarde hasta la puesta del sol, pero ocasionalmente, la faena se realiza durante todo el día, sobre todo cuando está nublado, para evitar la irradiación solar sobre los especímenes capturados. Eventualmente, los pescadores de alevinos utilizan redes de tipo mosquitero de $2 \mathrm{~mm}$ de malla que son operadas en la playa por cuatro a cinco pescadores que arrastran la red contra la orilla. 


\section{ALMACENAMIENTO POST CAPTURA}

Luego de la captura, los zúngaro tigrinus adultos son guardados en jaulas pequeñas de $1.5 \times 0.50 \times 0.50 \mathrm{~m}$, denominadas "rapisheo" por los pescadores locales, instaladas en la zona de orilla de los ríos, suspendidas sobre el fondo (Figura 4A). Los alevinos del zúngaro tigrinus son guardados en cajas de madera de $35 \times 35$ x $10 \mathrm{~cm}$, forradas de plástico que son utilizadas también, para el transporte a los centros de acopio que están localizados en la ribera o en la ciudad.

\section{TRANSPORTE AL ACUARIO COMERCIAL}

El transporte de los adultos, al acuario comercial, es realizado en cajas de madera de $1.5 \times 0.45 \times 0.30 \mathrm{~m}$, confeccionadas de palo de balsa, de baja densidad y poco peso. El número de peces por cada unidad de transporte depende del tamaño del pez. El transporte de los alevinos al acuario se realiza en bolsas plásticas, colocando un alevino en cada bolsa. Esta modalidad de transporte se practica con la finalidad de prevenir el canibalismo que, de otro modo, es muy acentuado (Figura 4B).

\section{NIVELES DEL RÍO YEPOCAS DE PESCA}

La captura de los zúngaro tigrinus, generalmente, es escasa, con uno o tres especimenes, como máximo, por faena de pesca. La captura está asociada al nivel del río. En el río Amazonas, tanto al estado de alevino, como de adulto, se realiza con mayor intensidad en vaciante que, corresponde, a los meses de julio a noviembre. En creciente, en especial, entre los meses de enero a abril, su captura es prácticamente nula, tanto porque la velocidad de la corriente en el río es mayor, como por el frecuente transporte de palizada que puede incluir árboles enteros, en la corriente del río, que dificultan la operación de las redes a la deriva y la maniobra de las embarcaciones de pesca.
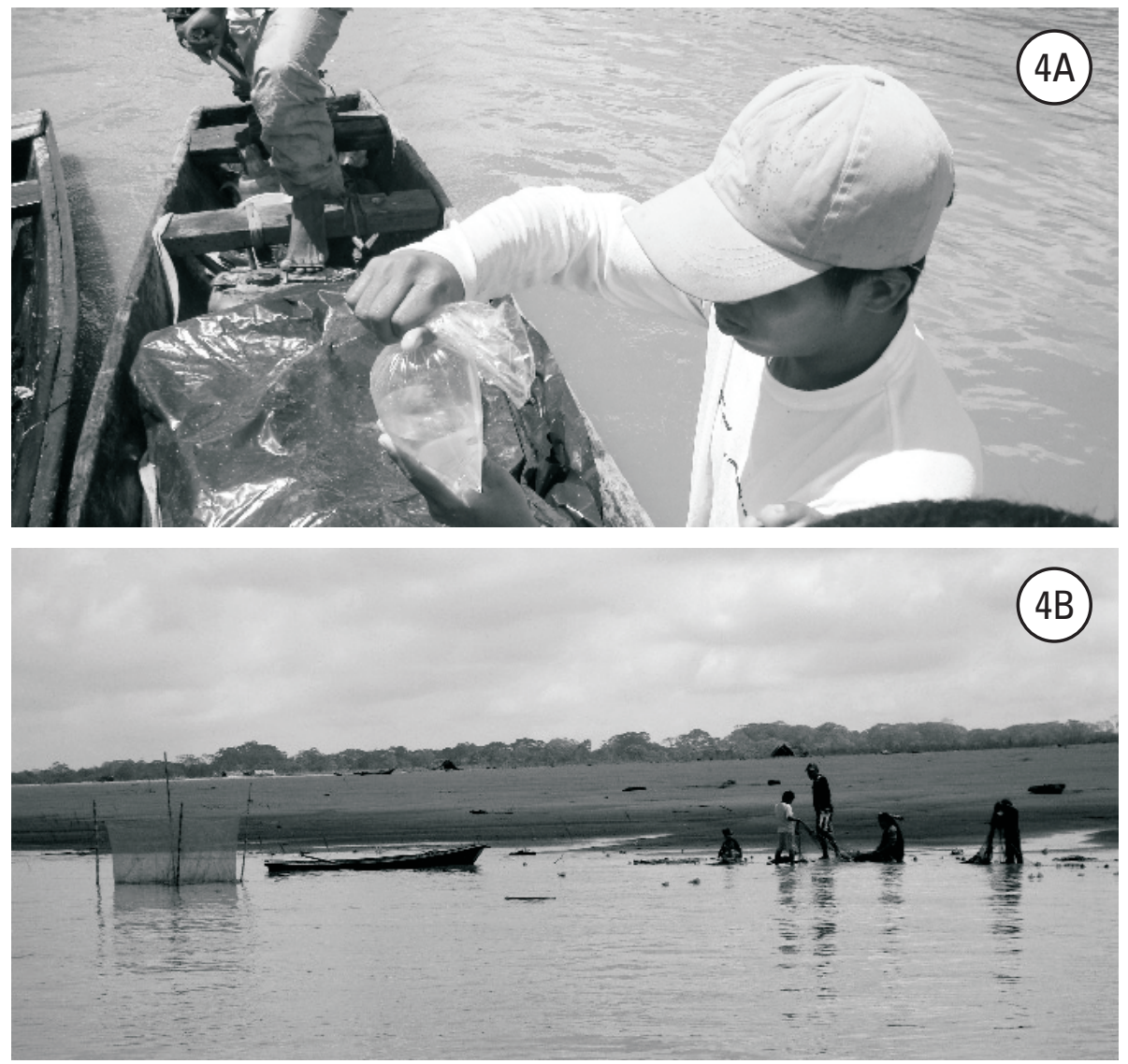

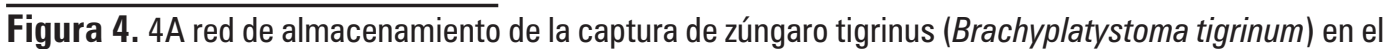
río Amazonas, 4B transporte de alevinos de zúngaro tigrinus desde los lugares de captura hasta los centros de acopio. 


\section{ALIMENTOS Y ALIMENTACIÓN}

El zúngaro tigrinus, como otros Pimelodidos, ocupa un alto lugar en la pirámide trófica; es decir, es un predador que, se alimenta de diversas especies de peces de porte pequeño conocidos como mojaras, los cuales captura en la columna de agua. Sin embargo, en un individuo, recientemente capturado se observó la regurgitación de una macana (Gymnotidae) en estado avanzado de digestión.

En condiciones de cría en ambiente controlado, al estado de alevino, el zúngaro tigrinus acepta peces de porte pequeño, como guppies y mojaras, así como fases larvales de dípteros (Chironomidae), producido en condiciones controladas. Murrieta (2009)

\section{REPRODUCCIÓN}

La época de reproducción corresponde al final de la vaciante y comienzos de la creciente, en el periodo comprendido entre junio a noviembre, que corresponde al periodo de retracción del río. En el área de Iquitos, se compró zúngaro tigrinus adultos recientemente capturados, sin que se haya observado adultos en maduración. Por otra parte, cabe la posibilidad que, al igual que en doncella Pseudoplatystoma fasciatum y tigre zúngaro $P$. tigrinum, la maduración del zúngaro tigrinus tenga una mayor frecuencia de ocurrencia al inicio de la expansión del ambiente acuático, como fue reportado por Núñez et al. (2005) en observaciones efectuadas en la cuenca de los ríos Mamoré e Iténez, en la Amazonía boliviana.

En esta época los alevinos tienen un tamaño de 2 a 5 cm de longitud promedio, Murrieta (2009) observó la compra venta de alevinos de estas tallas en el mes de noviembre en las proximidades de Iquitos.

\section{PREDADORES}

No se conoce los predadores de los zúngaro tigrinus, pero, es probable que sean presa de otros carnívoros, entre ellos, otros Pimelodidos de mayor porte.

\section{PATÓGENOS}

En situación de cautiverio el zúngaro tigrinus criado en estaques de agua negra en el Centro de Investigaciones de Quistococha, del Instituto de Investigaciones de la Amazonía Peruana, presentó parasitismo por Argulus pestifer en una prevalencia de $100 \%$ y una intensidad de parasitismo de 12 a 30 por individuo $(\mathrm{N}=8)$ Alcántara et al. (2008B). El parásito produce erosiones de la piel, en el techo de la región cefálica, disminuyendo la condición general de los peces que, poco a poco, dejan de alimentarse.

\section{RELACIÓN LONGITUD PESO YFACTOR DE CONDICIÓN}

Los zúngaro tigrinus presentan una relación longitud peso descrito por la ecuación $\mathrm{Wt}=0.0034433 \mathrm{Lt}^{3.19027953}$ y coeficiente de relación $r=0.98283632$ (Figura 5) establecido por Alcántara et al. (2008A) que indica, una tendencia de crecimiento de tipo isométrico (Ricker, 1975) y al mismo tiempo, presentan un factor de condición de $0.77 \pm 0.12$ que corresponde a la condición del soma, antes que a madurez sexual. Alcántara et al. (2008A). El cálculo de este factor, es de suma importancia porque permite estimar, por comparación, la condición real de los zúngaro tigrinus en condiciones de cría en el estanque de cultivo.

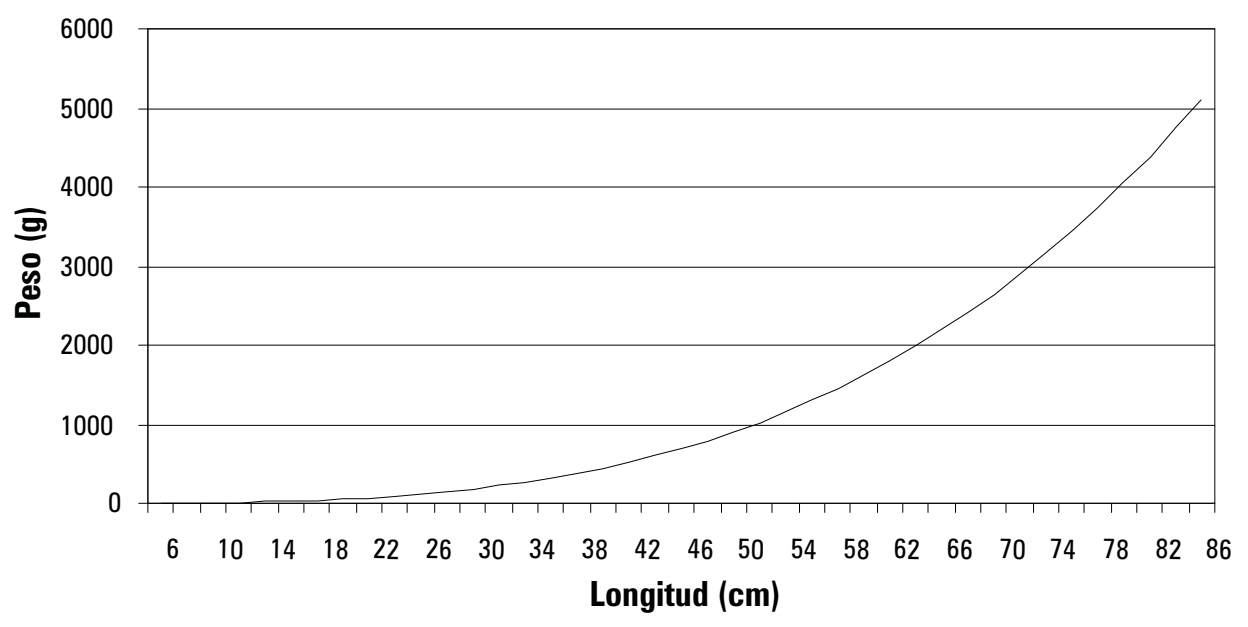

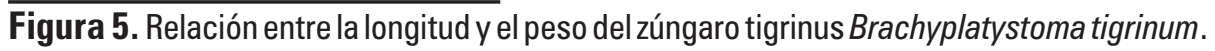




\section{SOCIOECONOMÍA}

Durante el período junio noviembre de 2009 los zúngaro tigrinus adultos alcanzaron un precio de $\mathrm{S} /$. 170.00, en el mercado de Iquitos, debido a su escasez en las capturas. A su vez, los zúngaro tigrinus, al estado de alevino, alcanzaron un precio, en el mismo periodo, de S/. 15.00 a S/. 35.00 por unidad. Si se tiene en cuenta que el jornal diario en Iquitos, fue de S/. 15.00, se deduce claramente, la importancia que tiene esta especie, en el mercado local, como fuente de ingresos para los pescadores de peces de consumo y de peces ornamentales que operan en el río Amazonas, en las inmediaciones de Iquitos.

Adicionalmente, esta especie tiene importancia, también como fuente de ingresos para las instalaciones comerciales mayoristas que, exportan los zúngaro tigrinus al estado de alevino y juvenil, a los centros de mayor demanda, como son los mercados de Asia y Europa, en donde puede alcanzar precios de más de U. S. \$. 150.00 por unidad, dependiendo del tamaño.

\section{BIBLIOGRAFÍA CITADA}

Alcántara, F., Chu-Koo, F., Rodríguez, L., Chávez, C., Tello, S. \& Núñez, J. 2008A. Relación longitudpeso y el factor de condición del zúngaro tigrinus Brachyplatystoma tigrinum del río Amazonas. Loreto. Perú. Folia Amazónica. Vol 17 No 1-2:2338.

Alcántara, F., Chu-Koo, F., Rodríguez, L., Chávez, C. Bernuy, A., Barbarán, T., Tello, S. \& Núñez, J. 2008B. Primer reporte de parasitismo de Brachyplatystoma tigrinum por Argulus pestifer en acuicultura. Folia Amazónica. Vol 17 No 12:99-102.

Asociación de pescadores de peces ornamentales de Loreto, Iquitos, APPOLI. 2009. Programa de Manejo Pesquero de Alevinos de zúngaro tigrinus Merodontotus zúngaro tigrinus y zúngaro alianza Brachyplatystoma juruense. Base legal D. S. No 015-2009 - PRODUCE. Iquitos. Perú. 30 p.
Chávez V. Carlos A., Alfonso Bernuy R., Teddy Barbarán R., Germán Murrieta M., Homero Sánchez R., Fred Chu K., Fernando Alcántara B., Jesús Nuñez. 2008. Reporte de los conocimientos tradicionales sobre el zúngaro tigrinus Brachyplatystoma tigrinum en la Amazonía peruana. Red de Investigación sobre la Ictiofauna Amazónica. En prensa. 6 p.

Moreau, Marie - Annick \& Cooms, O. 2007. Aquarium fish exploitation in western Amazonía: Conservation issues in Peru. Foundation for Environmental Conservation. Environmental Conservation 34 (1): 12-22.

Murrieta, G. 2009. Manejo de alevinos de zúngaro tigrinus Brachyplatystoma tigrinum (Britski, 1981) (Pisces, Pimelodidae) en condiciones controladas. Tesis para optar el titulo profesional de Biologo. Universidad Nacional de la Amazonia Peruana. $42 \mathrm{p}$.

Núñez, J., Inturias, A., Dugué, R., Barreto, J., Rivera, R., Dupponchelle, F., Renno, J. F. 2005. Reproducción y crecimiento de Pseudoplatystoma fasciatum en la cuenca amazónica Boliviana. En, Renno, J. F., García, C., Dupponchelle, F., Núñez, J., Editores. Biologia de las Poblaciones de Peces de la Amazonía y Piscicultura. 27 de junio - 1 de julio de 2005. Iquitos. Perú. Red de Investigación sobre la Ictiofauna Amazónica. RIIA. p 47-51.

Olivier, K. 2001. The ornamental fish market. FAO/Globefish Research Programme, Vol. 67. United Nations Food and Agriculture Organization, Rome, Italy.

Ricker, W. E. 1975. Computation and Interpretation of Biological Studies of Fish Population. Department of the Environment Fisheries and Marine Service. P203-212.

Sánchez, H. 2008. Reporte sobre la ictiofauna del alto Marañón. IIAP. Informe interno. 12 p. 